\title{
O USO DE CULTURA CLONAL DE PRECURSORES HEMATOPOIÉTICOS NA INVESTIGAÇÃO DO POTENCIAL MIELOSSUPRESSIVO DE NOVAS SUBSTÂNCIAS
}

\author{
The use of clonal culture of hematopoietic progenitors in the investigation of the \\ potential myelossuppresive of new chemicals
}

\author{
Marize C. Valadares \\ Faculdade de Farmácia-Universidade Federal de Goiás. E-mail: marizecv@farmacia.ufg.br
}

Recebido em 09/11/2204 - Aceito em 23/11/2004

\begin{abstract}
RESUMO: Este artigo é uma breve revisão bibliográfica sobre a aplicação dos ensaios de cultura clonal de precursores hematopoéticos, em particular o ensaio clonogênico de progenitores hematopoieticos para granulócitos e macrófagos ("Colony Forming Unit - Granulocyte Macrophage", CFU-GM) na investigação de mielotoxicidade.
\end{abstract}

PALAVRAS-CHAVE: CFU-GM, xenobióticos, medula óssea, toxicidade.

ABSTRACT: This article is a short review of the employment of the hematopoietic progenitor clonogenic assay, in particular the clonogenic assay for hematopoietic progenitors of granulocyte/macrophage ("Colony Forming Unit - Granulocyte Macrophage", CFU-GM) in the investigation of myelotoxicity.

KEYWORDS: CFU-GM, xenobiotics, bone marrow, toxicity.

\section{INTRODUÇAO}

A produção das células sanguíneas é um processo complexo envolvendo proliferação celular, diferenciação, morfogêneses, maturação funcional e morte celular (METCALF, 1984). As células-tronco hematopoéticas ("stem cell"), residentes na medula óssea, possuem a capacidade única de auto-renovação e diferenciação nos diferentes tipos de células sangüíneas. A partir da célula-tronco hematopoiética as células se comprometem com uma das três linhagens distintas: mielóide, linfóide ou eritróide em resposta aos fatores de crescimento humoral e citocinas específicas presentes no local (GRIBALDO, 2002; DIODOVICH, et al., 2004). Além dos precursores hematopoiéticos, a medula óssea é constituída também pelo estroma medular: células endoteliais, células acumuladoras de lipídios, macrófagos e células fibroblásticas, formando assim o microambiente hematopoiético (PARENT-MASSIM et al., 2001). O estroma medular tem como função a regulação da maturação e diferenciação dos precursores hematopoiéticos por meio de contato direto entre as células do estroma e células hematopoiéticas ou de forma indireta por meio da liberação dos fatores de crescimento. A integridade destes dois sistemas, bem como a associação entre eles, são fatores cruciais para a manutenção da homeostase hematopoiética (LORD \& TESTA, 1988; DELLMANN, 1993).

Devido à grande capacidade proliferativa do tecido hematopoiético, as células da medula óssea são alvos freqüentes da ação tóxica de xenobióticos (YAMAGUCHI et al., 1994). Desta forma, a avaliação da integridade da hematopoiese é um parâmetro importante para o entendimento dos mecanismos envolvidos na alteração da produção de células sanguíneas e como estas mudanças coletivamente irão impactar o sistema imunológico (DELDAR et al., 1988; PARENT-MASSIN et al., 1993; YAMAGUCHI et al., 1994; GRIBALDO et al., 1996; 
PARCHMENT 1998; GRIBALDO et al., 2000; NEGRO et al., 2001; PESSINA et al., 2001; GRIBALDO 2002; PESSINA et al., 2003; DIODOVICH et al., 2004).

Os ensaios clonogênicos, simulando a hematopoiese, são sistemas de cultura de células progenitoras hematopoiética, geralmente em meio semi-sólido, amplamente empregado na hematologia experimental e clinica por mais de 30 anos. Na hematologia clínica estes métodos são empregados para confirmar diagnóstico, em transplantes de medula óssea ou ainda para predizer o tempo de recuperação medular nos casos de falência deste órgão (DELDAR et al., 1988; DELDAR \& STEVENS, 1993; DELDAR, 1994; DELDAR, HOUSE \& WIERDA, 1995). Já na área experimental, estes ensaios são aplicados para se avaliar a hematopoiese frente às condições patológicas com o estresse, infecção bacteriana, câncer, bem como para avaliar o efeito de drogas, a exemplo, os agentes quimioterápicos, sobre a hematopoise, (VALADARES et al., 1998; MALACRIDA et al., 1997; DANTAS \& QUEIROZ 1999; QUEIROZ et al., 2001; VALADARES \& QUEIROZ 2002; QUEIROZ et al., 2004).

A utilização de ensaios clonogênicos de forma investigativa em estudos pre-clinicos de novas substâncias é mais recente. In vitro, em presença de meio de cultura para tecidos e de uma mistura de citocinas específicas para cada linhagem celular, os progenitores formam colônias distintas fenotipicamente de células diferenciadas. A estas células deu-se o nome de unidades formadoras de colônias ("Colony Forming Unit", CFU) (QUEIROZ, 1988). Os ensaios clonogênicos de progenitores aplicados às investigações hematotoxicológicas incluem unidades formadoras de colônia comprometida com a linhagem de granulócitos e macrófagos ("Colony Forming Unit-Granulocyte Macrophage", CFU-GM), com a linhagem eritróide ("Burst Forming Unit-Eritroid", BFU-E) e com a linhagem megacariocítica ("Colony Forming Unit-Megakaryocyte", CFU-MK) (PARENT-MASSIN 2001; MALERBA et al., 2004).

O tipo de hematotoxicidade mais freqüente e amplamente estudado in vitro são os efeitos agudos de drogas sobre os progenitores da medula óssea da série granulocítica/macrofágica. A quantificação da mielotoxicidade é feita á partir do número de células progenitoras sobreviventes em função do nível de exposição ao xenobiótico na presença de concentrações máximas do fator estimulador de colônias de granulócitos e macrófagos ("Granulocyte-Macrophage Colony Stimulating Factor" GM-CSF) (METCALF 1984; GRIBALDO 2002; PESSINA et al., 2003; DIODOVICH et al., 2004).

Uma vez que a mielotoxicidade pode resultar tanto do efeito direto da droga sobre os progenitores hematopoiéticos quanto do efeito sobre as células do estroma medular ou expressão de citocinas/receptores, diferente protocolos de investigação têm sido desenvolvidos e propostos para a avaliação da integridade da hematopoiese in vitro (PARENT-MASSIN et al., 1993; GRIBALDO et al., 1996; PESSINA et al., 1998; PESSINA et al., 1999; PESSINA et al., 2003). Todos estes testes são modificações da técnica original descrita por BRADLEY e METCALF (1966), e também desenvolvida e modificada por outros autores (DEXTER et al., 1973; DEXTER \& TESTA, 1976; DEXTER \& SPOONCER, 1987).

Além da cultura clonal de precursores hematopoiéticos para granulócitos e macrófagos (CFU-GM), o modelo mais estudado, merece citação a cultura líquida de longa duração da medula óssea ("Long Term Bone Marrow Culture", LTBMC). Esta cultura consiste em um modelo in vitro para estudo do estroma medular (GRIBALDO el al., 1999; GRIBALDO et al., 2000). A supressão da formação do estroma medular induzido por drogas leva a um declínio rápido da formação de CFU-GM e morte das células progenitoras. Um outro aspecto importante neste sistema é a capacidade metabólica das células estromais mediada por enzimas do citocromo P450, o que permite ainda a avaliação dos efeitos de metabólitos de drogas sobre a mielopoiese (MYERS \& FLESHER, 1990).

In vivo, substancias como os antineoplásicos, toxinas microbianas e radiação ionizante destroem rapidamente os progenitores da medula óssea em divisão, sendo que uma única dose aguda pode resultar em neutropenia ou trombocitopenia 4 a 20 dias após a exposição (NEELIS et al., 1997; MALERBA et al., 2002). Durante o estágio de desenvolvimento de uma nova droga, um aspecto importante é predizer se o agente novo será clinicamente tóxico para a medula óssea e se a toxicidade é específica para uma linhagem celular. A mielotoxocidade é o maior fator limitante para o uso das doses requeridas dos antineoplásicos no tratamento dos tumores. Desta forma, usualmente se busca predizer dois níveis de exposição: a maior dose que não causará efeitos adversos clínicos e qual a dose que causa o máximo de efeitos tóxicos reversíveis tolerados, denominado Máxima Dose Tolerada (MTD). O estabelecimento da MTD é importante na escolha das doses iniciais empregadas nos ensaios de Fase I do desenvolvimento de fármacos (PARCHMENT et al., 1998; PARCHMENT, 2000).

O Centro Europeu para a Validação de Métodos Alternativos ("European Centre for the Validation of Alternative Methods", ECVAM) em conjunto com uma rede de laboratórios internacionais realizaram a validação do método in vitro CFU-GM como uma importante ferramenta na investigação preditiva de neutropenia clinica durante a fase investigativa pré-clinica de novas substâncias. Neste ensaio, são empregadas células de medula óssea murina e/ou células humanas de cordão umbilical (ricas em "stem cell") (Figura 1). Os dados obtidos são: qual dose ou nível plasmático da droga será hematotóxico, qual a menor dose que produz citopenia, qual a menor dose em que mais provavelmente ocorrerá o restabelecimento celular, qual a máxima dose tolerada e potencial de novas substâncias produzirem neutropenia clínica (PARENT-MASSIN et al., 1994; GRIBALDO et al., 1999; 
PESSINA et al., 2003; DIODOVICH et al., 2004; MALERBA et al., 2004). Alem disso, nestes ensaios é possível demonstrar efeitos sinérgicos e antagônicos de vários compostos (DU et al., 1990). Desta forma é possível se obter informações da toxicologia/farmacologia humana, no caso do emprego de células de cordão umbilical, mesmo em estágios investigativos iniciais que poderão direcionar as próximas etapas da investigação toxicológico-terapêutica de novas drogas.

\section{Ensaio clonogênico CFU-GM}

No ensaio clonogênico CFU-GM aplicado à toxicologia, os progenitores hematopoiéticos, numa concentração celular conhecida, são semeados em placa de petri de $35 \mathrm{~mm}$ contendo meio semi-sólido, como por exemplo, agar $(0,3 \%)$ ou metilcelulose, na presença de citocinas apropriadas (SCF, GM-CSF e Interleucina-3) e o xenobiótico sob investigação, Figura 1 . As placas são incubadas a $37^{\circ} \mathrm{C}$, na presença de $5 \%$ de $\mathrm{CO}_{2}$, por 7 dias para células murinas ou 12-14 dias para células humanas. O número de colônias é então quantificado com o auxílio de microscópio de dissecção (PESSINA et al., 2002). Atualmente estuda-se um método automatizado para a contagem das colônias (MALERBA et al., 2004). Cada progenitor produz uma colônia. As colônias em cultura são originárias de uma única célula sendo, portanto, consideradas clonadas (PARENT-MASSIN, 2001). Uma vez que as células progenitoras são heterogêneas e considerando o tamanho do clone, a cinética de desenvolvimento e distribuição, a análise das diferenças de tamanho, na presença de um xenobiótico, permite a distinção entre as diferentes expressões do efeito tóxico. Os efeitos tóxicos podem se apresentar como: destruição das células, o bloqueio da mitose, atraso da mitose ou o aumento no número de mitoses. Utilizando curvas de dose-efeito é possível determinar como referência as concentrações inibitórias para $50 \%$ ou $90 \%$ de proliferação celular, ou seja, o IC ${ }_{50}$ ou IC 90 , respectivamente (PARENT-MASSIN, 2001; PESSINA et al., 2001).

A granulopoiese é a parte da hematopoiese responsável pela produção de granulócitos e macrófagos através da proliferação e diferenciação de progenitores específicos, CFU-GM (CD13 ${ }^{+}, \mathrm{CD}_{3} 3^{+}, \mathrm{CD}_{3} 4^{+}, \mathrm{CD} 115^{+}$, $\mathrm{CD}_{116^{+}}, \mathrm{CD}^{2} 3^{+}, \mathrm{CD}^{2} 131^{+}$) (PARENT-MASSIN, 2001).

A cultura CFU-GM foi inicialmente utilizada para confirmar a origem da agranulocitose. Isto foi realizado para demonstrar a relação entre desordens sanguíneas e a administração de drogas (LIND et al., 1973; BARRET et al., 1976; KELTON et al., 1979; IRVINE et al., 1994; ASCENSAO et al., 1984; PARENT-MASSIN et al., 1993). Estes autores mostraram que a realização sistemática da cultura de CFU-GM após o restabelecimento medular, na presença de drogas e/ou soro da fase aguda, poderia ser um critério adicional para a avaliação da possibilidade do envolvimento de drogas nos casos de agranulocitose, acompanhados após a utilização da droga.

Neste contexto, o desenvolvimento de ensaios preditivos in vitro para hematotoxicidade utilizando o ensaio clonal CFU-GM foi um grande avanço. Inicialmente estes ensaios eram realizados com células de medula óssea humana, e posteriormente foram feitos com células murinas com o objetivo de melhor viabilizar o método, torna-lo reproduzível, fácil, barato e que possa ser utilizado para avaliação de xenobióticos como pesticidas, contaminantes ambientais, alimentos e drogas novas (PARENT-MASSIN \& THOUVENOUT, 1993; GRIBALDO, 1998; PARENT-MASSIN, 2001).

Uma vez estabelecido os ensaios murinos, um outro grande passo foi o estabelecimento de testes utilizando células humanas de cordão umbilical para se obter dados estimando os níveis toleráveis que facilitariam a busca de agentes terapêuticos com grande potencial terapêutico e baixa toxicidade. Em 1994, PARCHMENT e colaboradores estabeleceram uma correlação entre a severidade clinica de neutropenia com o pirazoloacridina e a inibição de CFU-GM in vitro. Durante um Workshop da ECVAM em 1995, sobre a utilização dos ensaios in vitro para a avaliação da hematotoxicidade, os participantes elegeram como prioridade máxima a validação do método de CFU-GM como sendo preditivo de neutropenia, com base nas evidencias existentes relatadas para a sua utilização, bem como a similaridade entre os protocolos estabelecidos independentemente por vários laboratórios. Posteriormente, em um estudo de pré-validação com 6 drogas anticancer foi desenvolvido o procedimento operacional padrão (POP) para os ensaios de CFU-GM (PESSINA et al., 2001). Em 2003, PESINA e colaboradores publicaram a validação do método de CFU-GM para predizer neutropenia clinica e um modelo para predição da Máxima Dose Tolerada de xenobióticos mielosupressivos. Recentemente foi desenvolvida uma nova técnica miniaturizada realizada em placa de 96 poços para avaliação dos efeitos tóxicos de antineoplásicos sobre CFU-GM, a qual mostrou eficiência, alem de correlação com a técnica original realizada em placa de petri (MALERBA et al., 2004). 

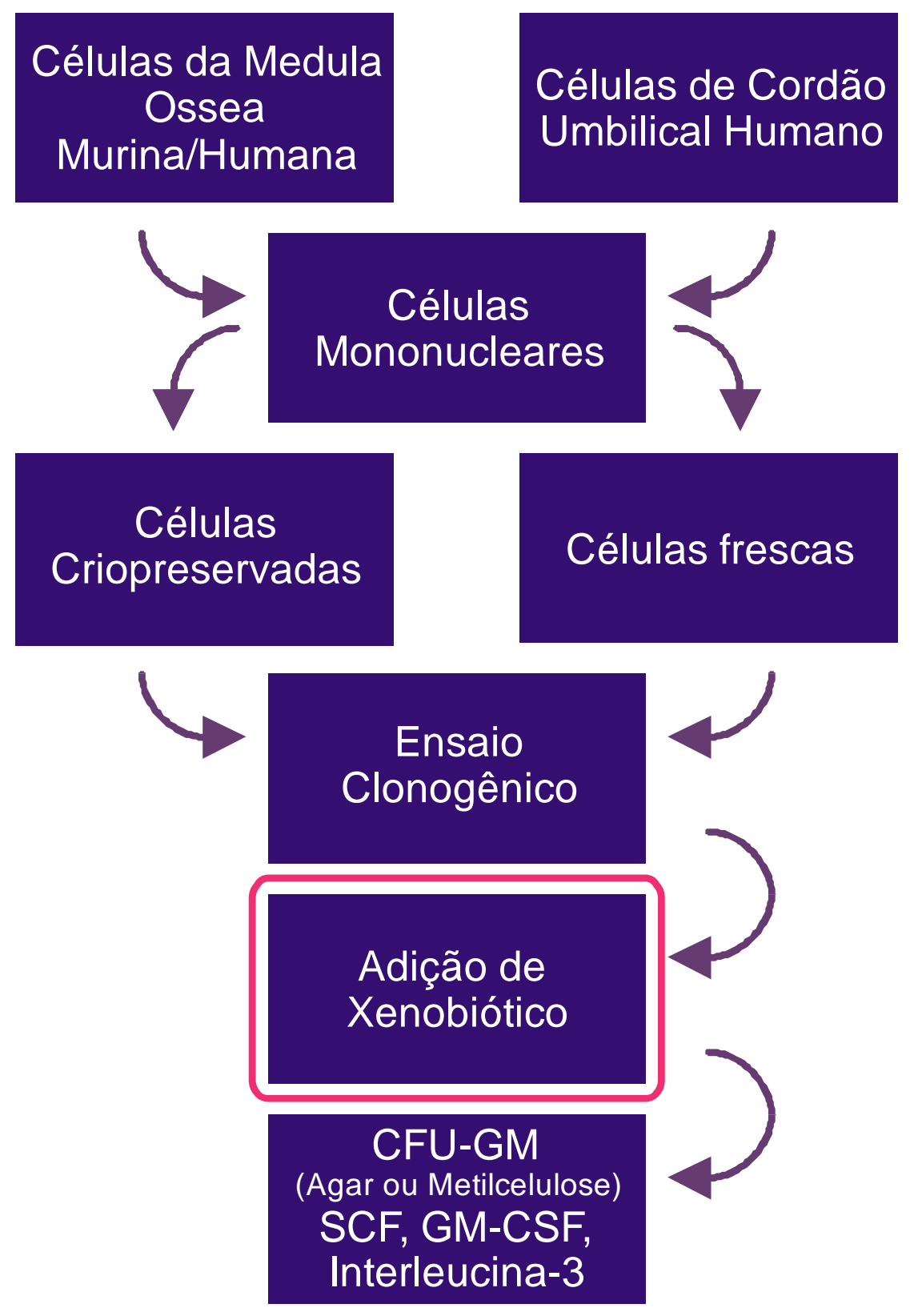

Figura 1: Ensaio clonogênico de CFU-GM em estudos toxicológicos.

\section{CONCLUSÕES}

O ensaio clonogênico de CFU-GM tem sido empregado para confirmar ou detectar o risco de um xenobiótico induzir danos á medula óssea, o que propicia a obtenção de informações toxicológicas humana nos estágios investigativos iniciais. Estes dados são utilizados para direcionar as próximas etapas da investigação toxicológica-terapêutica de novas drogas. A validação deste método pela ECVAM como preditivo de neutropenia clinica de agentes mielotóxicos consolidou o sucesso do método. Estudos com o objetivo de validar os métodos de CFU-MK e BFU-E estão em de andamento.

Além dos aspectos já mencionados, outra grande vantagem da aplicação deste método é a redução significativa do número de animais amplamente empregados na avaliação de toxicidade/segurança em investigações toxicológicas pré-clinicas. A desvantagem do método ainda é a contagem das colônias, devido ao número modesto de formação e a distribuição das colônias em diferentes níveis do meio de cultura.

Finalmente, é importante salientar ainda o desenvolvimento de novas metodologias capazes de elucidar o envolvimento de estruturas moleculares na citotoxicidade sobre os progenitores hematopoiéticos. 


\section{REFERENCIAS}

ASCENSAO, J.L.; FLYNN, P.J.; SLUNGAARD, A.; WACHSMAN, W.; ZANJANI, E.D.; JACOB, H.S. Quinidineinduced neutropenia: report of a case with drug-dependent inhibition of granulocyte colony generation. Acta Haematol.; V.72, n.5, p.349-54. 1984.

BARRET, A.J.; WELLER, E.; ROZENGURT, N.; LONGHURST, P.; HUMBLE, J.G. Amidopyrine agranulocitosis: drug inhibition of granulocyte colonies of patient's serum. Br Med J. V.ii, p. 850-1, 1976.

BRADLEY, \& METCALF, T.R. The growth of mouse bone marrow cells in vitro. Australian Journal of Experimental Biology and Medical Science, V. 40, p. 287-300, 1966.

DANTAS, D.C.; QUEIROZ, M.L. Effects of Chlorella vulgaris on bone marrow progenitor cells of mice infected with Listeria monocytogenes. Int J Immunopharmacol, V. 21, n.8, p. 499-508,1999.

DELDAR, A.; LEWIS, H.; BLOOM, J.; WEISS, L. Reproducible cloning assays for in vitro growth of canine hematopoietic progenitor cells and their potential applications in investigative hematotoxicity. Am J Vet Res, v. 49, n. 8, p.1393-40, 1988.

DELDAR, A. Drug-induced blood disorders: Review of pathogenetic mechanisms and utilisation of bone marrow cell culture technology as an investigative approach. Curr. Topics Vet. Res.,v.1,p. 83-101, 1994.

DELDAR, A.; HOUSE, V.R. WIERDA, D. Bone marrow colony-forming assays. Methods in Immunotoxicology, v.1, p. 227-250, 1995.

DELDAR, A., STEVENS, C. E. Development and application of in vitro models of Hematopoiesis to drug development. Toxicol. Path., V. 21, p. 231-240, 1993.

DELLMANN, H.D. Textbook of Veterinary Histology 4th edition, p. 66, Lea \& Febinger, Malvern, PA, 1993.

DEXTER, T. M.; ALLEN, T. D.; LAJTHA, L. G.; SCHOFIELD, R., LORD, B. I. Stimulation of differentiation and proliferation of hemopoietic cells in vitro. J. Cell. Physiol., v.82, p. 461-470, 1973.

DEXTER, T. M., SPOONCER, E. Growth and differentiation in the hemopoietic system. Ann. Rev. Cell. Biol., v.3, p. 423-441, 1987.

DEXTER, T. M. TESTA, N. G. Differentiation and proliferation of hemopoietic cells in culture. Meth. Cell. Biol. V. 14, p. 387-405. 1976.

DIODOVICH, C.; BIANCHI, M. G.; BOWE, G.; ACQUATI, F.; TARAMELLI, R.; PARENT-MASSIN, D.; GRIBALDO L. Response of human cord blood cells to styrene exposure: evaluation of its effects on apoptosis and gene expression by genomic technology. Toxicology. V., 5;n. 200, p. 45-57, 2004,

DU, D. L.; VOLPE, D. A.; GRIESHABER, C. K. MURPHY, M. J., J. R. Effects of L-phenylalaninemustard and Lbuthionine sulfoximine on murine and human hematopoietic cells in vitro. Cancer Res. V.,50,p.4038-4043, 1990.

GRIBALDO, L.; ALISON, M.; ANDREWS, P. W.; BREMER, S.; DONOVAN, P.J.; KNAAN-SHANZER, S.; MERTELSMANN, R.; SPIELMANN, H.; TESTA, N. G.; TRIFFITT, J. T.; ZIPORI, D.; DE WYNTER, E. Meeting summary: European Workshop on Stem Cells, European Centre for the Validation of Biomedical Testing Methods, Institute for Health and Consumer Protection, Joint Research Centre, Ispra, Italy, November 21-23, 2001. Exp Hematol, v. 30, n. 7, p. 628-33, 2002.

GRIBALDO, L.; CASATI, S.; CASTOLDI, A. F.; PESSINA, A. Comparison of in vitro drug-sensitivity of human granulocyte-macrophage progenitors from two different origins: umbilical cord blood and bone marrow. Exp Hematol, v.27, n, 11, p.1593-8, 1999. 
GRIBALDO, L.; CATALANI, P.; ERMINIO, M. Metabolism of doxorubicin in long-term bone marrow cultures and SR-4987 stromal established cell line. Drug Metabol Drug Interact, v. 15, n. 4, p. 279-91, 1999.

GRIBALDO, L.; MALERBA, I.; COLLOTTA, A.; CASATI, S.; PESSINA, A. Inhibition of CFU-E/BFU-E by 3'-azido3'-deoxythymidine, chlorpropamide, and protoporphirin IX zinc (II): a comparison between direct exposure of progenitor cells and long-term exposure of bone marrow cultures. Toxicol Sci, v. 58, n. 1, p.96-101, 2000.

GRIBALDO, L.; SACCO, M. G.; CASATI, S.; ZUCCHI, I.; DOSANJH, M.K.; CATALANI, P.; MARAFANTE, E. Modulation of proto-oncogene expression by polychlorinated biphenyls in 3T3-L1 cell line. J Toxicol Environ Health, v. 25, n.55, p. 121-31, 1998.

GRIBALDO, L. Haematotoxicology: scientific basis and regulatory aspects. Altern Lab Anim., v. 30, Suppl 2, p.1113,2002

GRIBALDO, L.; BUEREN, J.; DELDAR, P.; HOKLAND, P.; MEREDITH, C.; MONETA, D.; MOSESSO, P.; PARCHMENT, R.; PARENT-MASSIN, D.; PESSINA, A.; SAN ROMAN, J.; SCHOETERS, G. The use of in vitro systems for evaluating haematotoxicity. ECVAM Workshop Report 14. ATLA, v., 24, p. 211, 1996.

GRIBALDO, L.; MALERBA, I.; COLLOTTA, A.; CASATI, S.; PESSINA, A. Inhibition of CFU-E/BFU-E by 3'-azido-3'deoxythymidine, chlorpropamide, and protoporphyrin IX zinc (II): a comparison between direct exposure of progenitor cells and long-term exposure of bone marrow cultures. Toxicol Sci,v. 58, p. 96-101, $2000 .$.

IRVINE, A. E.; FRENCH, A.; DALY, A.; RANAGHAN, L.; MORRIS, T. C. Drug-induced neutropenia due to direct effects on CFU-C--ten years of culture experience. Eur J Haematol.,v. 52, n. 1, p. 21-7, 1994.

KELTON, J. G.; HUANG, A. T.; MOLD, N.; LOGUE, G.; ROSSE, W. F. The use of in vitro technics to study druginduced pancytopenia. N Engl J Med., v. 20, p. 621-4, 1979

LIND, D. E.; LEVI, J. A.; VINCENT, P. C. Amodiaquine-induced agranulocytosis: toxic effect of amodiaquine in bone marrow cultures in vitro. Br Med J. v. 1, p, 458-60, 1973.

LORD, B. I.; TESTA, N. G., The hemopoietic system: structure and regulation. In: N.G. Testa and R.P. Gale, Editors, Hemopoiesis: long-term effects of chemotherapy and radiation, Marcel Dekker, New York, p. 1-26, 1988.

MALACRIDA, S. A.; TEIXEIRA, N. A.; QUEIROZ, M. L. Hematopoietic changes in rats after inescapable and escapable shocks. Immunopharmacol Immunotoxicol, v. 19, n. 4, p. 523-33, 1997.

MALERBA, I.; CASATI, S.; DIODOVICH, C.; PARENT-MASSIN, D.; GRIBALDO, L. Inhibition of CFU-E/BFU-E and CFU-GM colony growth by cyclophosphamide, 5-fluorouracil and taxol: development of a high-throughput in vitro method. Toxicol In Vitro, v. 18, n. 3, p. 293-300, 2004.

MALERBA, I.; CASTOLDI. A. F.; PARENT-MASSIN, D.; GRIBALDO, L. In vitro myelotoxicity of Propanil and 3,4Dichloroaniline on murine and human CFU-E/BFU-E progenitors. Toxicol Sci, v. 69,n. 433-438, 2002.

METCALF, D. The basic biology of haematopoiesis. In: Metcalf, D., Editor, The haemopoietic colony stimulating factors, Elsevier, Amsterdam, p. 1-26, 1984.

MYERS, S. R.; FLESHER, J. W. Metabolism of the carcinogen 3-methylcholanthrene in human bone marrow preparations. Drug Metab Dispos., v.18, n. 5, p. 664-9, 1990.

NEELIS, K. G.; DUBBELMANN, J. D.; LUO, Q. L.; THOMAS, G. R.; EATON, D. L.; WAGEMAKER, G. Simultaneous administration of TPO and G-CSF after cytoreductive treatment of Rhesus monkeys prevent thrombocytopenia, accelerates platelet and red cell reconstitution, alleviates neutropenia, and promotes the recovery of immature bone marrow cells. Exp. Hematol., v. 25, p.1084-1093, 1997.

NEGRO, G. D.; BONATO, M.; GRIBALDO, L. In vitro bone marrow granulocyte-macrophage progenitor cultures in the assessment of hematotoxic potential of the new drugs. Cell Biol Toxicol. V. 17, n. 2, p. 95-105, 2001. 
PARCHMENT, R. E. Oncology clinical trials and in vitro prediction models for xenobiotic toxicology. Develop. Animal Vet. Sci., v. 31, p. 693-708, 2000.

PARCHMENT, R. E.; VOLPE, D. A.; LORUSSO, P. M.; ERICKSON-MILLER, C. L.; MURPHY, M. J.; J. R.; GRIESHABER, C. K. In vivo-in vitro correlation of myelotoxicity of 9-methoxypyrazoloacridine (NSC-366140, $P D 115934)$ to myeloid and erythroid hematopoietic progenitors from human, murine, and canine marrow. J Natl Cancer Inst., v. 16, p. 273-80, 1994.

PARCHMENT, R. E. Alternative testing systems for evaluating non-carcinogenic, hematologic toxicity. Environ. Health Perspect, v. 106, Suppl 2, p. 541-557, 1998.

PARENT-MASSIN, D.; THOUVENOT, D. In vitro study of pesticide hematotoxicity in human and rat progenitors. J Pharmacol Toxicol Methods, v. 30, n. 4, p 203-7., 1993.

PARENT-MASSIN, D. Relevance of clonogenic assays in hematotoxicology. Cell Biol. Toxicol., v. 17, p. 87-94, 2001.

PESSINA, A.; ALBELLA, B.; BAYO, M.; BUEREN, J.; BRANTOM, P.; CASATI, S.; CROERA, C.; GAGLIARDI, G.; FOTI, P.; PARCHMENT, R.; PARENT-MASSIN, D.; SCHOETERS, G.; SIBIRIL, Y.; VAN DEN HEUVEL, R.; GRIBALDO, L. Application of the CFU-GM assay to predict acute drug-induced neutropenia: an international blind trial to validate a prediction model for the maximum tolerated dose (MTD) of myelosuppressive xenobiotics. Toxicol Sci, v. 75, n. 2, p.355-67, 2003.

PESSINA, A.;ALBELLA, B.; BAYO, M.; BUEREN, J.; BRANTOM, P.; CASATI, S.; CROERA, C.; PARCHMENT, R.; PARENT-MASSIN, D.; SCHOETERS, G.; SIBIRI, Y.; VAN DEN HEUVEL, R.; GRIBALDO, L. In vitro tests for haematotoxicity: prediction of drug-induced myelosuppression by the CFU-GM assay. Altern Lab Anim, v. 30, Suppl. 2, p.75-9, 2002.

PESSINA, A.; ALBELLA, B.; BUEREN, J.; BRANTOM, P.; CASATI, S.; GRIBALDO, L.; CROERA, C.; GAGLIARDI, G.; FOTI, P.; PARCHMENT, R.; PARENT-MASSIN, D.; SIBIRIL, Y.; VAN DEN HEUVEL, R. Prevalidation of a model for predicting acute neutropenia by colony forming unit granulocyte/macrophage (CFU-GM) assay. Toxicol In Vitro, v. 15, n. 6, p. 729-40, 2001.

PESSINA A, PICCIRILLO M, MINEO E, CATALANI P, GRIBALDO L, MARAFANTE E, NERI MG, RAIMONDI A. Role of SR-4987 stromal cells in the modulation of doxorubicin toxicity to in vitro granulocyte-macrophage progenitors (CFU-GM). Life Sci. 1999, 65(5):513-23.

PESSINA, A.; ALBELLA, B.; BUEREN, J.; BRANTOM, P.; CASATI, S.; GRIBALDO, L.; CROERA, C.; GAGLIARDI, G.; FOTI, P.; PARCHMENT, R.; PARENT-MASSIN, D.; SIBIRIL, Y.; VAN DEN HEUVEL, R. Prevalidation of a model for predicting acute neutropenia by colony forming unit granulocyte/macrophage (CFU-GM) assay. Toxicol. In Vitro, v. 15, p. 729-740, 2001.

PESSINA, A.The granulocyte macrophage colony-forming unit assay. In Animal Cell Culture Techniques (M. Clynes, Ed.), p. 217-230. Springer-Verlag, Berlin, 1998.

QUEIROZ, M. L. S.; VALADARES, M. C. ; BINCOLETTO, C.. Ehrlich ascites tumor as a tool in the development of compounds with immunomodulatory properties. Immunopharmacol Immunotoxicol, in press, 2004.

QUEIROZ, M. L.; JUSTO, G. Z.; VALADARES, M. C.; PEREIRA-DA-SILVA, F. R.; MULLER, A. H. Adjuvant effect of Pluchea quitoc extract on the resistance of tumor-bearing mice by modulation of the host hematopoietic response. Immunopharmacol Immunotoxicol, v. 23, n. 2, p. 215-28, 2001.

QUEIROZ, M. L. S. Células progenitoras hematopoiéticas em cultura- revisão bibliográfica. Ciência e Cultura, v. 40, p. 421-426, 1988. 
VALADARES, M. C.; KLEIN, S. I.; ZYNGIER, S.; QUEIROZ, M. L. Growth and differentiation of bone marrow hematopoietic cells in mice bearing Ehrlich ascite tumor and treated with Dicyclopentadienildichlorotitanium (IV). Int J Immunopharmacol, v. 20, n. 10, p. 573-81, 1998.

VALADARES, M. C.; QUEIROZ, M. L. The effect of a Titanocene Dichloride derivative, Ti IV (C5H5)(2) NCS(2), on the haematopoietic response of Ehrlich tumour-bearing mice. Eur J Pharmacol., v. 29; n. 439, p. 35-42, 2002.

YAMAGUCHI, F.; FURUHAMA, K.; MIYAMOTO, M.; SAGARA-ISHIJIMA, N.; TAKAYAMA, S. Application of hematopoietic progenitor assays for the estimation of hematotoxicity in rats. J Pharmacol Toxicol Methods, v. 31, $\mathrm{n}$. 2, p. 71-7, 1994. 